\title{
Protective Effect of Carvacrol against Paclitaxel-Induced Ototoxicity in Rat Model
}

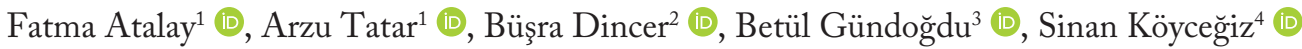 \\ ${ }^{1}$ Department of Otorhinolaryngology, Atatürk University School of Medicine, Erzurum, Turkey \\ ${ }^{2}$ Department of Pharmacology, Erzincan Binali Ylldırım University School of Pharmacy, Erzincan, Turkey \\ ${ }^{4}$ Clinic of Otorhinolaryngology, Mareşal Çakmak State Hospital, Erzurum, Turkey
}

Original Investigation $>{ }^{3}$ Department of Pathology, Atatürk University School of Medicine, Erzurum, Turkey

\begin{abstract}
Objective: This study aimed to explore whether carvacrol (CV) had a protective effect on paclitaxel-induced ototoxicity from biochemical, functional, and histopathological perspectives.

Methods: Forty Wistar albino male rats were randomly separated into five groups of eight rats. Group 1 was the control group, so Paclitaxel or CV was not administered. Group 2 was administered i.p. $\mathrm{CV}$ at 25 $\mathrm{mg} / \mathrm{kg}$ once a week; Group 3, was administered i.p. paclitaxel at $5 \mathrm{mg} / \mathrm{kg}$ once a week; Group 4 was administered i.p. paclitaxel at $5 \mathrm{mg} / \mathrm{kg}$ followed (30 min later) by CV at $25 \mathrm{mg} / \mathrm{kg}$ once a week; and Group 5 was administered i.p. CV at $25 \mathrm{mg} / \mathrm{kg}$ followed (1 day later) by paclitaxel at $5 \mathrm{mg} / \mathrm{kg}$. once a week. The drugs were administered intraperitoneally once a week for four consecutive weeks, and distortion product otoacoustic emissions (DPOAE) tests were performed at the beginning of the study before the first drug
\end{abstract}

ORCID iD of the author:

F.A. 0000-0002-0344-1982;

A.T. $0000-0002-4486-2695$;

B.D. 0000-0002-3365-7741;

B.G. 0000-0002-3786-3286

S.K. 0000-0002-9596-6646.

Cite this article as: Atalay $F$, Tatar $A$, Dincer B, Gündoğdu B, Köyceğiz S. Protective Effect of Carvacrol against Paclitaxel-

Induced Ototoxicity in Rat Model. Turk Arch

Otorhinolaryngol 2020; 58(4): 241-8.

\section{Corresponding Author:}

Fatma Atalay; fatalay@kastamonu.edu.tr

Received Date: 26.06 .2020

Accepted Date: 13.10 .2020

Content of this journal is licensed under a Creative Commons Attribution 4.0 International License. Available online at www.turkarchotolaryngol.net

\section{Introduction}

Ototoxicity refers to cellular degeneration and functional impairment of the cochlear and/or vestibular tissues and can lead to transient or permanent hearing loss $(1,2)$. Presently, drug-induced hearing loss is more frequently reported in clinical findings, especially in patients who have been treated with chemotherapeutic drugs (3). Today, there are no routine procedures or agents recommended for the treatment of chemotherapeutic agent-induced ototoxicity (2).

Paclitaxel, a taxane plant product isolated from Taxus brevifolia, is a broad-spectrum antineoplastic agent $(4,5)$. The damages the agent inflicts on the sensory neurons in the dorsal root ganglia administration and at the end of the study after the last drug administration. All rats were sacrificed, and cochleae were removed for biochemical and histopathological analysis.

Results: Biochemical data indicated that paclitaxel caused oxidative stress in the cochlea. Histopathological findings revealed the loss of outer hair cells in the organ of Corti $(\mathrm{CO})$ and moderate degenerative changes in the stria vascularis (SV). It was observed that DPOAE measurements were significantly reduced at high frequencies. In groups which $\mathrm{CV}$ was administered together with paclitaxel, these biochemical, histopathological, and functional changes were favorably reversed.

Conclusion: $\mathrm{CV}$ may have a protective effect against paclitaxel-induced ototoxicity when given.

Keywords: Carvacrol, ototoxicity, oxidative stress, paclitaxel, animal experimentation raises questions regarding its effects on peripheral auditory neurons. A few clinical studies were reported on the sensorineural hearing loss effects of paclitaxel (6). Although some researchers have observed the ototoxic effects of some anti-cancer agents such as cisplatin, which is especially well documented, research on the direct ototoxic effects of paclitaxel on the hair cells in the cochlea are limited $(2,4)$. The reason for the difficulty in understanding the ototoxic effect of paclitaxel may be due to its use in combination with other antineoplastic drugs, such as cisplatin, for which ototoxic effects were documented (7).

Evidence suggests that chemotherapeutic agents cause ototoxicity as a result of the accumulation of 
reactive oxygen species (ROSs) such as superoxide and hydroxyl radicals, and the depletion of glutathione (GSH) and antioxidant enzymes $(8,9)$. Therefore, the use of various antioxidant agents has been the strategy of numerous studies to prevent and restore the ototoxic effects of chemotherapeutic drugs $(10,11)$.

One of the agents with antioxidant properties is carvacrol $(\mathrm{CV})$, which is a monoterpenic phenol. It is found in the essential oils of many aromatic plants, including, among others, oregano, and thyme. CV is responsible for a wide range of pharmacologic activities, including antimicrobial, antioxidant, and anti-cancer activities (12). Remarkably, CV has high antioxidant activity, and numerous studies have investigated its antioxidant properties (13). Although $\mathrm{CV}$ is known to be a primary antioxidant, its role in paclitaxel-induced ototoxicity is unknown, and to the best of our knowledge, no previous studies were reported on this subject.

This study investigated whether CV had protective efficacy against the ototoxicity induced by paclitaxel, from biochemical, functional, and histopathological perspectives.

\section{Methods}

\section{Animals}

Forty Wistar albino male rats (weighing 250-280 g) were obtained from the Atatürk University Experimental Animal Laboratory of Medicinal and Experimental Application and Research Center. The care and handling of animals were in line with the principles of the Animal Ethical Committee of Atatürk University (Approval Date: August 22, 2016; Approval Number: 42190979-000-E. 1600192974). The rats were kept in standard plastic cages with ad libitum access to standard rat chow and tap water. Until the end of the study, the cages were kept in a room equipped with an air-conditioner set at $22 \pm 1^{\circ} \mathrm{C}$ and a fully automated lighting system (12 h light/12 h dark).

\section{Chemicals}

The CV used in this study was obtained from Sigma-Aldrich (W224511; Sigma-Aldrich Chemical Company; Taufkirchen, Germany). The selected dose of CV was $25 \mathrm{mg} / \mathrm{kg}$ (14) and administered intraperitoneally (i.p.) as calculated per weight for each rat.

\section{Main Points}

- Ototoxicity is the functional disorder of the cochlear and / or vestibular structures, which is frequently reported in clinical findings, due to the use of chemotherapeutic drugs.

- Carvacrol $(\mathrm{CV})$ is a monoterpene phenol known for its powerful antioxidant properties.

- Based on the data from the rat cochleae, this study showed that CV might have a protective effect on paclitaxel-induced ototoxicity from biochemical, functional, and histopathological perspectives.

- The study suggests that CV may be useful as a preventive option in patients receiving paclitaxel against possible ototoxicity.
Paclitaxel was purchased from Actavis Pharma (Sindaxel; Actavis Drug Co., İstanbul, Turkey) and administered i.p. at $5 \mathrm{mg} /$ $\mathrm{kg}$, as calculated per weight for each rat.

Finally, ketamine hydrochloride was obtained from Pfizer (Ketalar; Pfizer Drug Co., İstanbul, Turkey), and xylazine from Bioveta (Xylazinbio; Bioveta, Ankara, Turkey). An anesthetic mixture was prepared with $10 \mathrm{mg} / \mathrm{kg}$ xylazine, and $40 \mathrm{mg} /$ $\mathrm{kg}$ ketamine hydrochloride, administered i.p. as calculated per weight to each rat for anesthesia.

\section{Experimental Design}

At the beginning of the study, all rats were anesthetized intraperitoneally with an anesthetic mixture $(50 \mathrm{mg} / \mathrm{kg}$ ketamine hydrochloride- $10 \mathrm{mg} / \mathrm{kg}$ xylazine) to perform otoscopic examination and measure distortion product otoacoustic emissions (DPOAE). Rats with a pathology detected in the outer ear canal and tympanic membrane, and whose hearing was not normal were excluded from the study. The study consisted of five groups, namely the control group and four experimental groups. Each group contained eight randomly selected rats. The sample size of the study was determined according to similar studies previously conducted $(5,15,16)$.

Group 1 - Control group: $1 \mathrm{~mL}$ serum physiologic was administered i.p. once a week for four consecutive weeks.

Group 2 - CV group: CV was administered i.p. at $25 \mathrm{mg} / \mathrm{kg}$ once a week for four consecutive weeks.

Group 3 - Paclitaxel group: Paclitaxel was administered i.p. at 5 $\mathrm{mg} / \mathrm{kg}$ once a week for four consecutive weeks.

Group 4 - Paclitaxel + CV group (pre-treatment carvacrol): first a $5-\mathrm{mg} / \mathrm{kg}$ dose of paclitaxel was given and $30 \mathrm{~min}$ later $25-\mathrm{mg} /$ $\mathrm{kg}$ of $\mathrm{CV}$ was administered i.p. once a week for four consecutive weeks.

Group 5 - CV + Paclitaxel group (post-treatment carvacrol): first $25 \mathrm{mg} / \mathrm{kg} \mathrm{CV}$ was given and 1 day later $5 \mathrm{mg} / \mathrm{kg}$ paclitaxel was administered i.p. once a week for four consecutive weeks.

The second DPOAE measurements were taken under general anesthesia one day later after the last drug application. The rats were sacrificed to perform the experiments; their temporal bones were dissected, and cochleae were removed. Right cochleae were used for histopathological studies and the left cochleae for biochemical analysis.

\section{Otoacoustic Emissions Measurements}

The DPOAE measurements were performed with an Otometrics MADSEN Capella device (Otometrics MADSEN Capella Otoacoustic emissions testing; Natus Medical Denmark ApS) using a suitable probe. The first DPOAE measurements were performed under general anesthesia, after otoscopic examination, and before drug administration in a silent room (in the first week). The second DPOAE measurements were performed af- 
ter the last drug application (in the fourth week). Measurements were recorded in the distortion product gram (DPgram) form. In the DPgram measurement, primary stimulus intensities were equalized at $65 \mathrm{~dB}(\mathrm{~L} 1=\mathrm{L} 2)$. The two separate frequencies (f1 and f2) were arranged as $\mathrm{f} 2 / \mathrm{f} 1=1.22$ to receive the strongest responses. Furthermore, the DPgram measurements were taken at $996 ; 1191 ; 1416 ; 1679 ; 2001 ; 2382 ; 2832 ; 3359 ; 4003 ; 4755$; 5654; 6728; and $7998 \mathrm{~Hz}$. In the DPOAE measurements, values equal to $3 \mathrm{~dB}$ and higher for the signal-to-noise ratio (SNR) were evaluated as positive.

\section{Biochemical Investigation of Cochleae}

After the left cochleae were removed, cochlear tissues were stored at $-80^{\circ} \mathrm{C}$ until analysis. These tissues were set in liquid nitrogen with a Tissue Lyser II (Qiagen; Hilden, Germany) grinding jars set. All cochlear specimens (about $100 \mathrm{mg}$ of tissue) were homogenized in $1 \mathrm{~mL}$ of phosphate-buffered saline (PBS) homogenate buffer. Then, to obtain the supernatant, homogenized tissues were centrifuged, and superoxide dismutase (SOD) activity was measured as described in the literature (17). Values were expressed as units per milligram of protein. GSH levels were determined using the methods described by Sedlak and Lindsay (18) and expressed as nanomoles per milligram of protein. Malondialdehyde (MDA) levels were also measured using a technique described in the literature (19). Concentrations were expressed as nanomoles per milligram of protein, and SOD activity, as well as GSH and MDA levels were measured at room temperature with an enzyme-linked immunosorbent assay (ELISA) reader.

\section{Histopathological Evaluation of Cochleae}

After the right cochleae were harvested for histopathological analysis, they were fixed for $24 \mathrm{~h}$ in $10 \%$ buffered formaldehyde. Then, decalcification was done in these tissues with $6 \%$ nitric acid. After decalcification was completed, the decalcification solution was washed out under running water for approximately 30 minutes. After routine processes, tissues were embedded in paraffin blocks. These were then sectioned at $5 \mu \mathrm{m}$ thickness. Sections were transferred onto glass slides and stained with hematoxylin and eosin (H\&E). The sections of the cochlear tissues were evaluated under a light microscope by pathologists who were blind to the grouping of samples. Presence of Corti organ (CO) damage was evaluated based on grading system of de Freitas et al. (20), and the grading of $\mathrm{CO}$ damage was assessed based on the number of external ciliated cells (ECCs) following de Freitas et al.'s (20) 4-point scoring system:

0: presence of three ECCs with intact nuclei;

1: presence of two ECCs with intact nuclei;

2: presence of one ECC with intact nuclei;

3: loss of CO.

The status of the stria vascularis (SV) was evaluated based on Yazici et al.'s (21) staging system. Staging was assessed based on the degree of cell shrinkage.

According to the staging scoring system, tissues were categorized as follows:
$0=$ no damage;

$1=$ slight damage;

$2=$ moderate damage;

$3=$ serious damage.

\section{Statistical Analysis}

Statistical analysis was performed using the IBM Statistical Package for the Social Sciences v20.0 (IBM SPSS Corp.; Armonk, NY, USA) software. While evaluating the DPOAE results, compatibility with normal distribution was evaluated with the Kolmogorov-Smirnov test. The paired t-test was used to compare the groups before and after the administration in terms of normally distributed data. Data were presented as mean \pm standard deviation, and $p<0.05$ was considered statistically significant. Histopathological findings were analyzed with the Kruskal-Wallis test and significance was accepted at $\mathrm{p}<0.001$. Analyses between two groups were performed with the Mann-Whitney U test with Bonferroni correction. When evaluating the results of the biochemical analysis, comparisons between the groups were made with the one-way ANOVA and Tukey's tests; significance was accepted at $\mathrm{p}<0.05$.

\section{Results}

\section{Results of DPOAE Measurements}

The DPOAE measurements were taken before and after drug administration at 3000, 4000, 6000 and $8000 \mathrm{~Hz}$. Amplitude changes in groups were compared for both the right ear and the left ear (Figure 1). There were no statistically significant differences in pre- and post-treatment DPOAE measurements at any frequency $(3000-8000 \mathrm{~Hz})$ in Groups 1, 2, 4 and 5 (p>0.05). Also in Group 3, no statistically significant differences were observed in the pre- and post-treatment DPOAE measurements at frequencies of 3000 and $4000 \mathrm{~Hz}$ ( $p>0.05$ ), but statistically significant decrease were detected in the post-treatment measurements at $6000-$ and $8000-\mathrm{Hz}$ frequencies $(\mathrm{p}<0.05)$.

\section{Results of Biochemical Parameters}

The results of the GSH and MDA levels and SOD activity of rat cochleae are shown in Figure 2. In Group 3 MDA levels $(p=0.000)$ were significantly increased, while SOD activity $(\mathrm{p}=0.000)$ and GSH levels $(\mathrm{p}=0.000)$ were significantly decreased compared to Group 1. Compared to Group 3, statistically significant increases were found in the SOD activity ( $\mathrm{p}=0.005, \mathrm{p}=0.007, \mathrm{p}=0.006$ respectively) of Groups 2,4 , and 5 . Compared to Group 3, statistically significant increases were found in the GSH levels $(0.002,0.009,0.020$ respectively) of Groups 2, 4, and 5, whereas statistically significant decreases were found in the MDA levels of Groups of 2 ( $\mathrm{p}=0.005), 4$ $(\mathrm{p}=0.006)$, and $5(\mathrm{p}=0.002)$ compared to Group 3. In other words, the level of GSH and the SOD activity in cochlear tissue had significantly decreased in the paclitaxel group, whereas in the CV groups, high GSH levels and SOD activity values were found. Moreover, MDA levels, which are lipid peroxidation indicators, were increased by paclitaxel, while CV prevented this increase with an antioxidative effect and remained close to the control group. 
Table 1. Histopathological scoring results of $\mathrm{CO}$ and $\mathrm{SV}$ damage

\begin{tabular}{l|c|c|c|c|c|c|}
\hline & Group 1 & Group 2 & Group 3 & Group 4 & Group 5 & p \\
\hline CO damage & $0(0-0)$ & $0(0-0)$ & $3(3-3)^{*}$ & $0(0-0)$ & $0(0-0)$ \\
\hline SV damage & $0(0-0)$ & $0(0-1)$ & $2(2-2)^{*}$ & $0(0-0)$ & $1(1-1)$ & $<0.001$ \\
\hline
\end{tabular}

The Kruskal-Wallis test was used for statistical comparisons and $\mathrm{p}<0.001$ was considered significant. The paclitaxel group (Group 3 ) was compared with the other groups, ${ }^{*} \mathrm{p}<0.001$ marks were used.

CO: corti organ; SV: stria vascularis

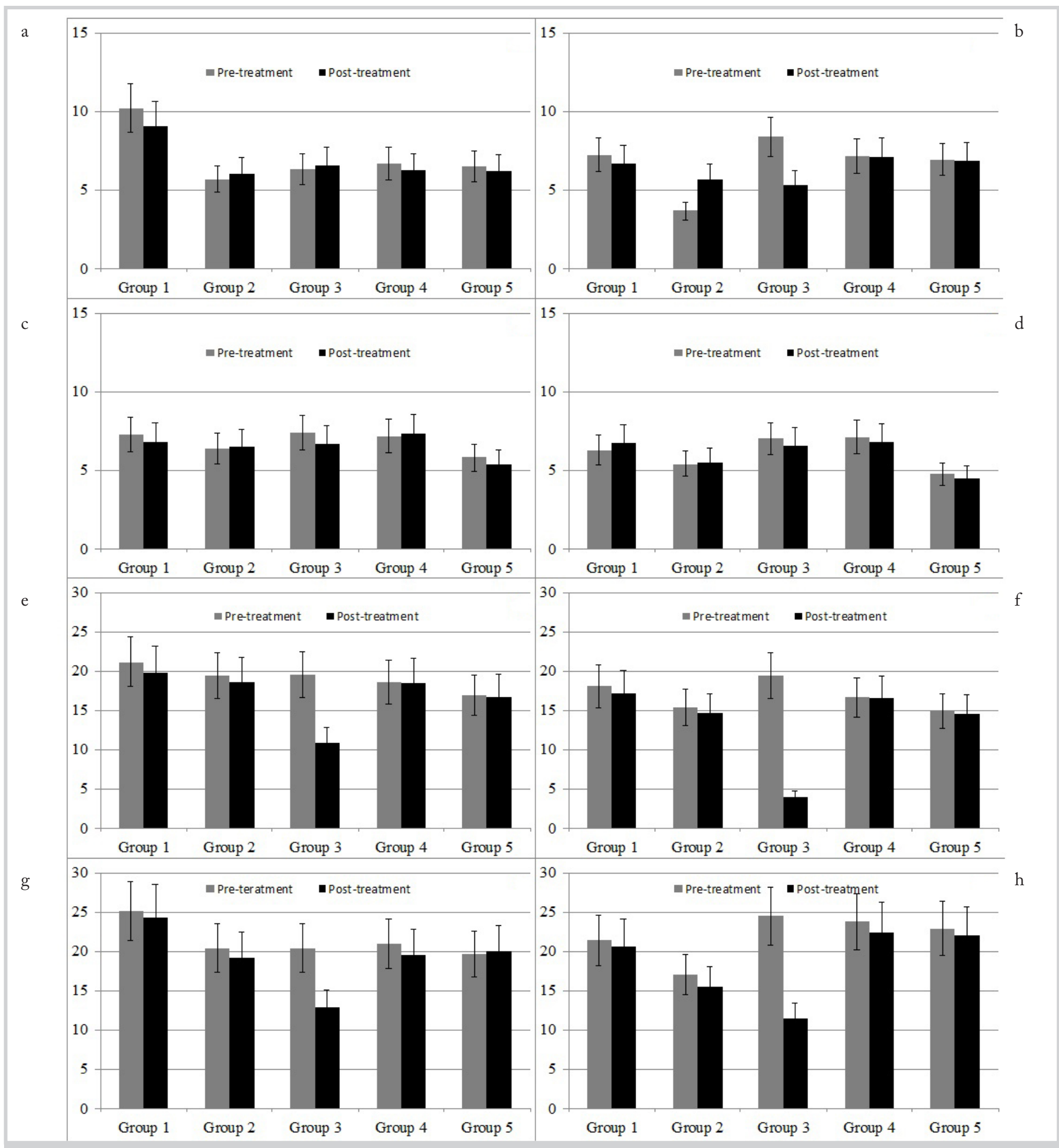

Figure 1. a-h. Average DPOAE amplitudes pre and post drug treatment for each group. (a) at $3000 \mathrm{~Hz}$ frequency (right ear); (b) at $3000 \mathrm{~Hz}$ frequency (left ear); (c) at $4000 \mathrm{~Hz}$ frequency (right ear); (d) at $4000 \mathrm{~Hz}$ frequency (left ear); (e) at $6000 \mathrm{~Hz}$ frequency (right ear); (f) at 6000 $\mathrm{Hz}$ frequency (left ear); (g) at $8000 \mathrm{~Hz}$ frequency (right ear); (h) at $8000 \mathrm{~Hz}$ frequency (left ear) 

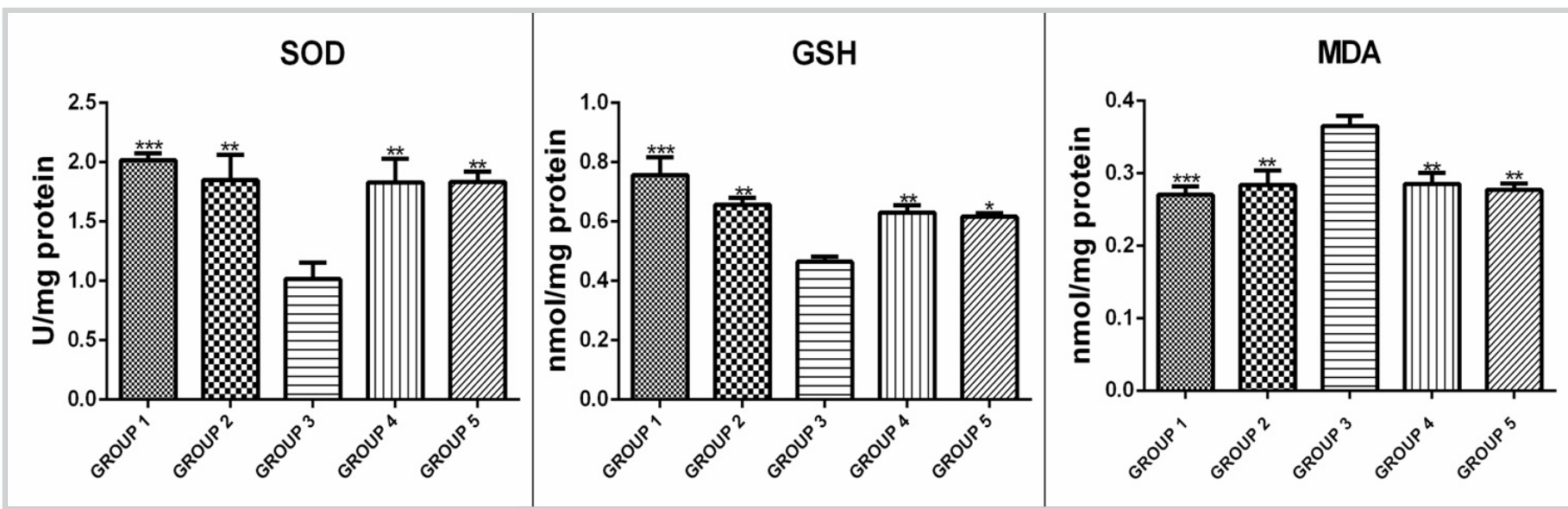

Figure 2. a-c. (a) SOD activity, (b) GSH and (c) MDA levels of the groups subjected to paclitaxel-induced ototoxicity Statistical comparisons were made using one-way ANOVA followed by Tukey's test. The paclitaxel group was compared with the other groups, *p<0.05, *** $\mathrm{p}<0.01$, and ${ }^{* * * *} \mathrm{p}<0.001$ marks were used. Values are represented as mean $\pm \mathrm{SD}$

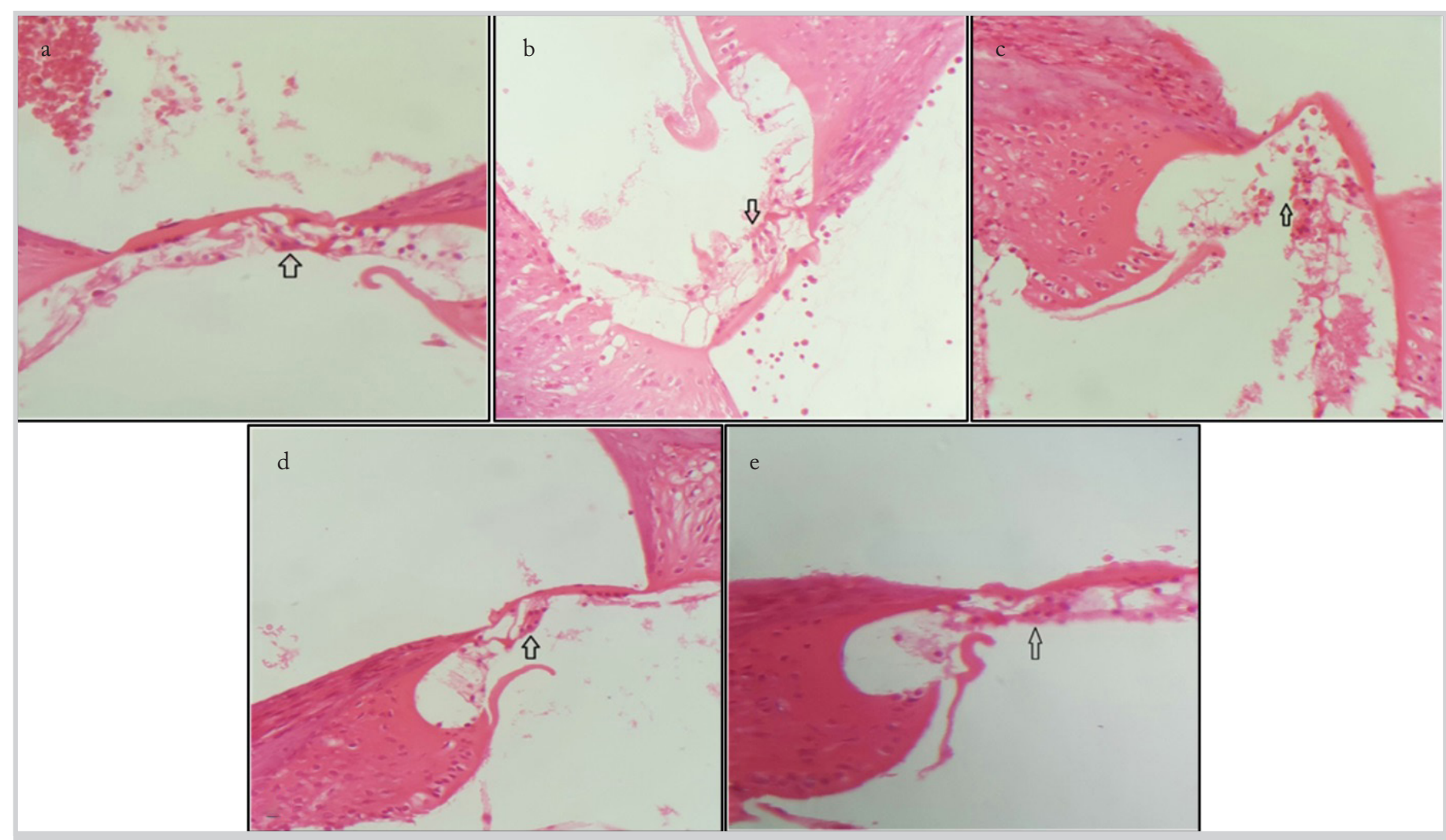

Figure 3. a-e. Stria vascularis sections of all groups. (a) Group 1, (b) Group 2, (c) Group 3, (d) Group 4, (e) Group 5. Dye: H\&E Magnification: $\mathrm{x} 400$

\section{Results of Histopathological Evaluation}

Histopathological scoring results were evaluated for $\mathrm{CO}$ according to the grading system described by de Freitas et al. (20) and for SV damage according to the staging system described by Yazici et al. (21). These are presented in Table 1. In the groups $2,4,5, \mathrm{CO}$ and SV damage scoring was lower than in Group 3 , which was the paclitaxel group $(\mathrm{p}<0.001)$. Histopathological examination of sections taken from cochleae were obtained using $H \& E$ staining under a light microscope (Figures 3, 4). The histopathological appearance of the cochleae exhibited normal architecture in Groups 1, 2, and 4. Examination of the cochleae in Group 3, however, found significant degeneration character- ized by moderate degenerative changes in the SV (Figure 3) and loss of outer hair cells in CO (Figure 4). Finally, in Group 5, outer hair cells were close to a normal histopathologic appearance; however, mild degeneration in SV were observed.

\section{Discussion}

This is the first study that investigated the protective effect of $\mathrm{CV}$ in a paclitaxel-induced ototoxicity model. The role of $\mathrm{CV}$ in ototoxicity was observed by investigating its effect on oxidative stress parameters. The data were also supported with findings from histopathologic changes. Although paclitaxel is a widely used chemotherapeutic agent in the treatment of various cancers 


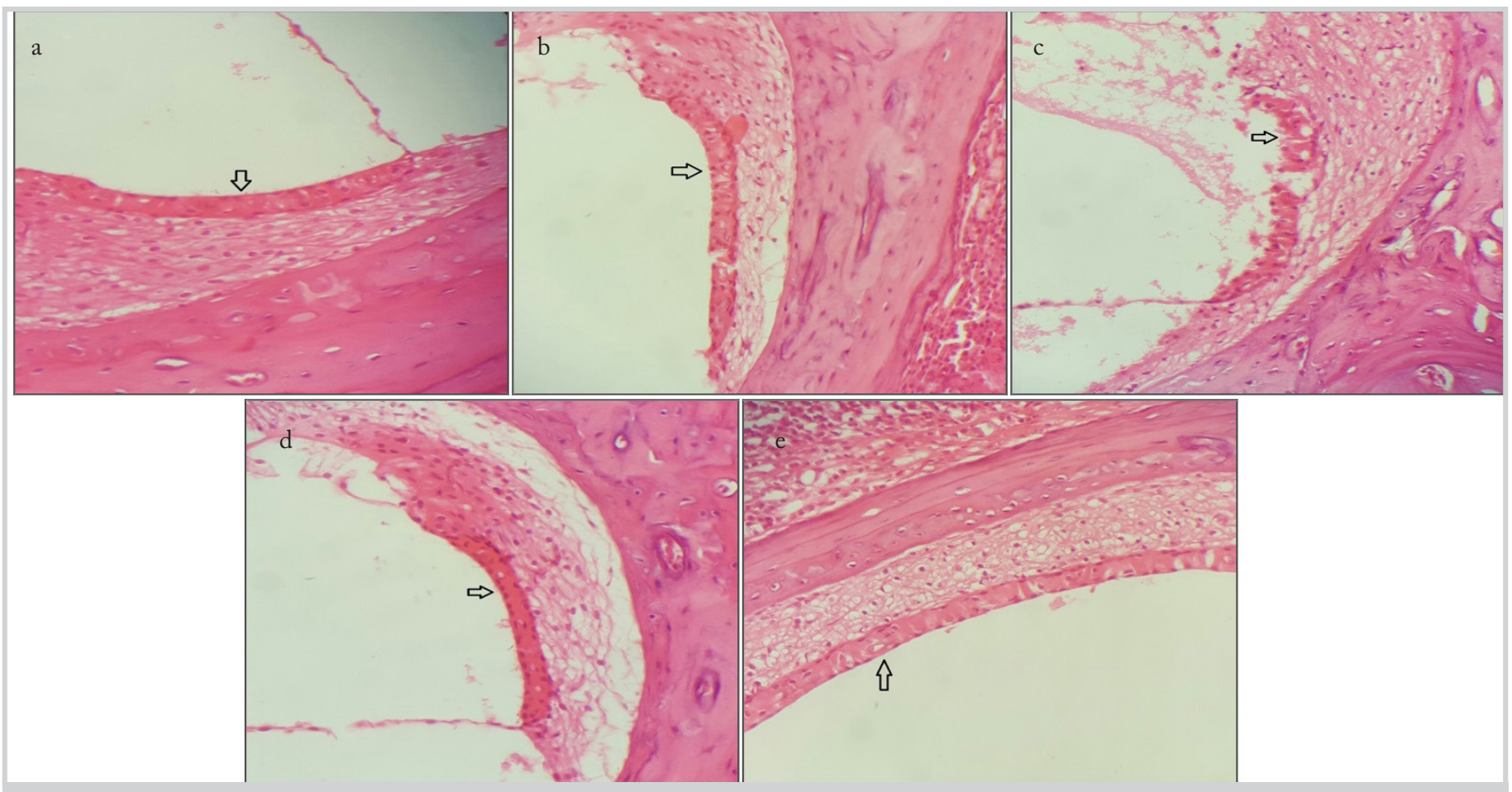

Figure 4. a-e. Corti organ sections of all groups. (a) Group 1, (b) Group 2, (c) Group 3, (d) Group 4, (e) Group 5. Dye: H\&E Magnification: x400

$(22,23)$, there is not much research yet on whether paclitaxel has an ototoxic effect, because paclitaxel is used with other chemotherapeutic agents known to have ototoxic effects (7). This situation conceals the possible ototoxic effects of paclitaxel. Also, whether CV has protective effect on ototoxicity has not been studied to date. Therefore, our study is deemed to contribute to the literature by investigating both the effect of paclitaxel-which is used in the treatment of many cancers-and the effect of $\mathrm{CV}$ - which may be a new candidate for the treatment of ototoxicity. The fact that a recent study has already shed light on paclitaxel's ototoxic effects, demonstrates that more studies should be conducted on this subject (5).

Today, there is no routine treatment method and agent used to prevent ototoxicity. The molecular mechanism of chemotherapeutic-drug-related ototoxicity has not been fully elucidated. However, numerous studies exist on the prevention and repair of ototoxicity (2). The basis of these studies is that chemotherapeutic drugs cause ototoxicity due to the uncontrolled increase in ROSs (9). Excessive production of ROSs results in the depletion of GSH and the accumulation of superoxide ions, leading to the inhibition of antioxidant enzymes in the cochlea. Depletion of the antioxidant defense system then causes an increase in lipid peroxidation. The generation of ROSs consequently activates apoptosis and causes cell damage (24). Therefore, antioxidant agents that both prevent the uncontrolled production of ROSs and strengthen the antioxidant defense system are widely used to counteract the ototoxic effect of chemotherapeutics. Several studies report to have used antioxidants such as pomegranate, gallic acid, vitamin $\mathrm{E}$, and curcumin to prevent ototoxicity (5, $10,11,21)$. The presented study aimed to investigate whether CV's known antioxidant properties would have a protective effect on paclitaxel-induced ototoxicity.
$\mathrm{CV}$ is a monoterpenic phenol found in the essential oils of many aromatic plants such as, Thymus vulgaris, Origanum vulgare, Origanum majorana, and Citrus uranium bergama. It offers a wide range of biological effects that are useful in clinical practice, such as antibacterial, antioxidant, anti-cancer, antifungal, and antiviral properties. It is also worth noting that $\mathrm{CV}$ is well known for its strong antioxidant properties compared to other essential volatile oils. The strong radical-scavenging activity of $\mathrm{CV}$ was proven in both in vitro and in vivo studies (12). It demonstrated a hepatoprotective effect in a hepatotoxicity study by increasing the activity of enzymatic (superoxide dismutase, catalase, and GSH peroxidase) and non-enzymatic (vitamin $\mathrm{C}$ and E) antioxidants (25). Further, $\mathrm{CV}$ was observed to prevent lipid peroxidation and increase the endogenous antioxidant defense mechanism in a study on hepatocellular carcinogenesis (26). It was also demonstrated to improve acute pancreatitis with its strong antioxidant effect (13). Whereas the strong antioxidant effects of $\mathrm{CV}$ have been shown in some studies, there are no reports in the literature on the use of $\mathrm{CV}$ for ototoxicity. To the best of our knowledge, our study is the first to examine the effects of $\mathrm{CV}$ on ototoxicity.

In the presented study, an increase in MDA levels, a decrease in GSH levels, and SOD activity were observed in paclitaxel-induced ototoxicity (Group 3). CV exhibited an otoprotective effect by preventing ROS formation and repairing oxidative stress damage (Groups 4 and 5). Histological findings were observed as $\mathrm{CO}$ and $\mathrm{SV}$ damage in the paclitaxel-treated group (Group 3). In CV-treated groups (Groups 4 and 5), the degeneration caused by paclitaxel improved, and the cochleae had a normal histological appearance.

DPOAE measurements were performed in the first and fourth weeks to support our biochemical and histopathologic findings. 
The DPOAE test is a fast, inexpensive, non-invasive, and objective method that is frequently used in experimental studies to determine any damage in the cochlea (27). Based on the DPOAE measurements, significant decrease was observed in the fourth week at 6000 and $8000 \mathrm{~Hz}$ in the paclitaxel-administered group (Group 3) compared to the control group (Group 1). However, the decrease observed in the paclitaxel-administered group (Group 3) increased with the effect of CV (Groups 4 and $5)$ in DPOAE measurements and contributed to results comparable to that of the control group, hence was no significant difference between the before and after measurements. These results suggest that $\mathrm{CV}$ has an otoprotective role against paclitaxel-induced ototoxicity. The biochemical, histopathologic, and DPOAE results of the only $\mathrm{CV}$ - administered group (Group 2) were not statistically different from the control group. These results indicate the safety of $\mathrm{CV}$ in terms of ototoxicity.

This study has clear limitations that warrant consideration. Ototoxicity mainly affects high frequencies, which cannot be completely examined using the DPOAE measurements. Nevertheless, in this study, we observed the DPOAE results up to 8000 Hz. Another limitation is that we could not identify whether the protective effect of $\mathrm{CV}$ on a cochlear function to paclitaxel-induced ototoxicity is dose-dependent.

\section{Conclusion}

Considering the results which we obtained in rat cochleae, we can say that CV could play a protective role against paclitaxel-induced ototoxicity by lowering elevated ROSs and raising antioxidant enzyme levels. Our results are also supported by DPOAE measurements and histopathological findings, which suggest that $\mathrm{CV}$ may be useful as a preventive option in patients receiving paclitaxel against possible ototoxicity. Nevertheless, further studies are needed to determine the most appropriate doses and the indications of $\mathrm{CV}$ in clinical use.

Ethics Committee Approval: Ethics committee approval was received for this study from the Animal Ethical Committee of Atatürk University (Approval Date: August 22, 2016; Approval Number: 42190979000-E. 1600192974).

Peer-review: Externally peer-reviewed.

Author Contributions: Concept - F.A., A.T., B.D., B.G., S.K.; Design - F.A., A.T., B.D., B.G., S.K.; Supervision - A.T.; Resources - F.A., A.T.; Materials - A.T.; Data Collection and/or Processing - F.A., B.D.; Analysis and/or Interpretation - A.T., B.D., B.G., S.K.; Literature Search - F.A., B.G., S.K.; Writing - F.A., B.D.; Critical Reviews - F.A., A.T., B.D., B.G., S.K.

Conflict of Interest: The authors have no conflicts of interest to declare.

Financial Disclosure: The authors declared that this study has received no financial support.

\section{References}

1. Ganesan P, Schmiedge J, Manchaiah V, Swapna S, Dhandayutham S, Kothandaraman PP. Ototoxicity: a challenge in diagnosis and treatment. J Audiol Otol 2018; 22: 59-68.
2. Lanvers-Kaminsky C, Zehnhoff-Dinnesen AA, Parfitt R, Ciarimboli G. Drug-induced ototoxicity: mechanisms, pharmacogenetics, and protective strategies. Clin Pharmacol Ther 2017; 101: 491-500.

3. Landier W. Ototoxicity and cancer therapy. Cancer 2016; 122: 1647-58.

4. Dong Y, Ding D, Jiang H, Shi JR, Salvi R, Roth JA. Ototoxicity of paclitaxel in rat cochlear organotypic cultures. Toxicol Appl Pharmacol 2014; 280: 526-33.

5. Bucak A, Ozdemir C, Ulu S, Gonul Y, Aycicek A, Uysal M, et al. Investigation of protective role of curcumin against paclitaxel-induced inner ear damage in rats. Laryngoscope 2015; 125: 1175-82.

6. Tibaldi C, Pazzagli I, Berrettini S, De Vito A. A case of ototoxicity in a patient with metastatic carcinoma of the breast treated with paclitaxel and vinorelbine. Eur J Cancer 1998; 34: 1133-4.

7. Ding D, He J, Allman BL, Yu D, Jiang H, Seigel GM, et al. Cisplatin ototoxicity in rat cochlear organotypic cultures. Hear Res 2011; 282: 196-203.

8. Gonçalves MS, da Silveira AF, Murashima AAB, Rossato M, Hippolito MA. Otoprotection mechanisms against oxidative stress caused by cisplatin. Int Arch Otorhinolaryngol 2020; 24: 47-52.

9. Prasad KN, Bondy SC. Increased oxidative stress, inflammation, and glutamate: potential preventive and therapeutic targets for hearing disorders. Mech Ageing Dev 2020; 185: 111191.

10. Hakiminia B, Goudarzi A, Moghaddas A. Has vitamin E any shreds of evidence in cisplatin-induced toxicity. J Biochem Mol Toxicol 2019; 33: 22349.

11. Kilic K, Sakat MS, Ekinci Akdemir FN, Yildirim S, Saglam YS, Askin S. Protective effect of gallic acid against cisplatin-induced ototoxicity in rats. Braz J Otorhinolaryngol 2019; 85: 267-74.

12. Sharifi-Rad M, Varoni EM, Iriti M, Martorell M, Setzer WN, del Mar Contreras M, et al. Carvacrol and human health: a comprehensive review. Phytother Res 2018; 32: 1675-87.

13. Bakır M, Geyikoglu F, Colak S, Turkez H, Bakır TO, Hosseinigouzdagani M. The carvacrol ameliorates acute pancreatitis-induced liver injury via antioxidant response. Cytotechnology 2016; 68: 1131-46.

14. Hakimi Z, Salmani H, Marefati N, Arab Z, Gholamnezhad Z, Beheshti F, et al. Protective effects of carvacrol on brain tissue inflammation and oxidative stress as well as learning and memory in lipopolysaccharide-challenged rats. Neurotox Res 2020; 37: 96576.

15. Özdemir D, Özgür A, Kalkan Y, Terzi S, Tümkaya L, Yılmaz A, et al. The protective effects of whortleberry extract against cisplatin-induced ototoxicity in rats. Braz J Otorhinolaryngol 2019; 85: 55-62.

16. Gündoğdu R, Erkan M, Aydın M, Sönmez MF, Vural A, Kökoğlu $\mathrm{K}$, et al. Assessment of the effectiveness of quercetin on cisplatin-induced ototoxicity in rats. J Int Adv Otol 2019; 15: 229-36.

17. Sun Y, Oberley LW, Li Y. A simple method for clinical assay of superoxide dismutase. Clin Chem 1988; 34: 497-500.

18. Sedlak J, Lindsay RH. Estimation of total, protein-bound, and nonprotein sulfhydryl groups in tissue with Ellman's reagent. Anal Biochem 1968; 25: 192-205.

19. Ohkawa H, Ohishi N, Yagi K. Assay for lipid peroxides in animal tissues by thiobarbituric acid reaction. Anal Biochem 1979; 95: 351-8.

20. de Freitas MR, de Castro Brito GA, de Carvalho JV Jr, Gomes RM Jr, Barreto Martins MJ, de Albuquerque Ribeiro R. Light microscopy study of cisplatin-induced ototoxicity in rats. J Laryngol Otol 2009; 123: 590-7. 
Atalay et al.

21. Yazici ZM, Meric A, Midi A, Arınc YV, Kahya V, Hafız G. Reduction of cisplatin ototoxicity in rats by oral administration of pomegranate extract. Eur Arch Otorhinolarynogol 2012; 269: 45-52.

22. Misiukiewicz K, Gupta V, Bakst R, Posner M. Taxanes in cancer of the head and neck. Anticancer Drugs 2014; 25: 561-70.

23. Jeansonne DP, Koh GY, Zhang F, Kirk-Ballard H, Wolff L, Liu $\mathrm{D}$, et al. Paclitaxel-induced apoptosis is blocked by camptothecin in human breast and pancreatic cancer cells. Oncol Rep 2011; 25: 1473-80.

24. Jung HH, Chang J, Yang JY, Choi J, Im GJ, Chae SW. Protective role of antidiabetic drug metformin against gentamicin induced apoptosis in auditory cell line. Hear Res 2011; 282: 92-6.
25. Aristatile B, Al-Numair KS, Veeramani C, Pugalendi KV. Effect of carvacrol on hepatic marker enzymes and antioxidant status in d-galactosamine-induced hepatotoxicity in rats. Fundam Clin Pharmacol 2009; 23: 757-65.

26. Jayakumar S, Madankumar A, Asokkumar S, Raghunandhakumar S, Gokula dhas K, Kamaraj S, et al. Potential preventive effect of carvacrol against diethylnitrosamine-induced hepatocellular carcinoma in rats. Mol Cell Biochem 2012; 360: 51-60.

27. Abdala C, Visser-Dumont L. Distortion product otoacoustic emissions: a tool for hearing assessment and scientific study. Volta Rev 2001; 103: 281-302. 